\title{
Premotor Correlates of Integrated Feedback Control for Eye-Head Gaze Shifts
}

\author{
Pierre A. Sylvestre and Kathleen E. Cullen \\ Aerospace Medical Research Unit, Department of Physiology, McGill University, Montreal, Quebec, Canada, H3G 1Y6
}

\begin{abstract}
Simple activities like picking up the morning newspaper or catching a ball require finely coordinated movements of multiple body segments. How our brain readily achieves such kinematically complex yet remarkably precise multijoint movements remains a fundamental and unresolved question in neuroscience. Many prevailing theoretical frameworks ensure multijoint coordination by means of integrative feedback control. However, to date, it has proven both technically and conceptually difficult to determine whether the activity of motor circuits is consistent with integrated feedback coding. Here, we tested this proposal using coordinated eye-head gaze shifts as an example behavior. Individual neurons in the premotor network that command saccadic eye movements were recorded in monkeys trained to make voluntary eye-head gaze shifts. Head-movement feedback was experimentally controlled by unexpectedly and transiently altering the head trajectory midflight during a subset of movements. We found that the duration and dynamics of neuronal responses were appropriately updated following head perturbations to preserve global movement accuracy. Perturbation-induced increases in gaze shift durations were accompanied by equivalent changes in response durations so that neuronal activity remained tightly synchronized to gaze shift offset. In addition, the saccadic command signal was updated on-line in response to head perturbations applied during gaze shifts. Nearly instantaneous updating of responses, coupled with longer latency changes in overall discharge durations, indicated the convergence of at least two levels of feedback. We propose that this strategy is likely to have analogs in other motor systems and provides the flexibility required for fine-tuning goal-directed movements.
\end{abstract}

Key words: saccades; gaze; eye- head coordination; feedback control; vestibular; head-unrestrained; pontine reticular formation

\section{Introduction}

A longstanding question in motor control research is "How does the brain generate accurate multijoint movements?" In 1967, Bernstein made the critical observation that consecutive repetitions of a motor task are characterized by "repetition without repetition," because each attempt is achieved by unique, nonrepetitive motor patterns. The concept of optimal feedback control has provided an interesting explanation for variable yet successful control of limb movement (for review, see Todorov, 2004). In this theoretical framework, the brain does not enforce the details of a specific movement trajectory or pattern of muscle activity. Instead, feedback is used to optimize acquisition of the goal of a movement. As a result, trajectory variability is readily allowed, whereas external perturbations to the limb are automatically corrected in-flight.

Significant trial-to-trial differences are also observed in the coordinated eye-head movements that are routinely used to rap-

Received Sept. 27, 2005; revised Feb. 13, 2006; accepted March 24, 2006.

This work was supported by the Canadian Institutes of Health Research, the Natural Sciences and Engineering Research Council of Canada, and Le Fonds de la Recherche en Santé de Québec. We thank Jefferson E. Roy for his contribution to the setup and initial experiments and Sofiane Cherif for his contributions to the analysis procedures. We would also like to express our gratitude to Daniel Guitton, Soroush Sadeghi, Ariana Andrei, Marion Van Horn, and Jessica Brooks for critically reading this manuscript. We thank Walter Kucharski and John Knowles for outstanding technical expertise.

Correspondence should be addressed to Kathleen E. Cullen, Aerospace Medical Research Unit, 3655 Promenade Sir William Osler, Montreal, Quebec, Canada, H3G 1Y6. E-mail: kathleen.cullen@mcgill.ca.

D01:10.1523/JNEUROSCI.4099-05.2006

Copyright $\odot 2006$ Society for Neuroscience $\quad$ 0270-6474/06/264922-08\$15.00/0 idly shift the axis of gaze in space (Freedman and Sparks, 1997; Sylvestre et al., 2002). Two general classes of models have emerged that can account for the control of eye-head gaze shifts. In the first, a single controller is used to minimize gaze error rather than enforce a specific eye or head trajectory (Scudder et al., 2002; Guitton et al., 2003), making it conceptually analogous to optimal feedback control. Alternatively, it has been proposed that a gaze displacement command is decomposed early on to control separate eye and head comparators (Phillips et al., 1999; Sparks, 1999; Freedman, 2001; Quessy and Freedman, 2004). In this schema, it is the individual displacements of the eye and head, rather than the overall accuracy of the movement per se, that are under feedback control.

To date, the available evidence has proven equivocal with respect to these two model classes. The finding that movement accuracy is preserved when head trajectories are experimentally altered during gaze shifts is readily accounted for by gaze comparator models (Laurutis et al., 1986; Guitton and Volle, 1987; Cullen et al., 2004). However, models based on separate comparators can also simulate this finding with the incorporation of neck reflexes (Freedman, 2001). Likewise, the responses of saccadic brainstem neurons during gaze shifts (Cullen et al., 1993; Cullen and Guitton, 1997; Phillips et al., 1999) have been reconciled with both single and separate comparator models (for review, see Sparks 1999).

Thus, a fundamental question remains: how are the motor commands to the eye and head musculature coordinated to en- 
sure accurate gaze shifts? Here, we recorded the responses of individual neurons in the brainstem saccadic premotor circuit during gaze shifts in which the head movements were unexpectedly altered midflight. If the gaze displacement command is decomposed to drive completely independent premotor feedback circuits, perturbations to the head should have no impact on the neuronal responses. In contrast, perturbations to the head would produce substantial changes in activity if gaze accuracy is ensured via feedback to an integrated gaze controller. We observed significant changes in the response of saccadic premotor neurons, providing evidence that an integrated eye-head controller is used to ensure accuracy during gaze shifts.

\section{Materials and Methods}

Three monkeys (two Macaca mulatta, one Macaca fascicularis) were used in this study. During the experiments, the monkey was seated in a primate chair that was fixed to the suprastructure of a vestibular turntable. The monkey was trained to orient for a juice reward to a small red target light that was projected onto an isovergent monochromatic screen surrounding the turntable. Single-unit recording procedures, eye and head position monitoring using the magnetic search coil technique, target display, and data acquisition procedures have been described previously (Sylvestre and Cullen, 1999). All procedures have been approved by the McGill University Animal Care Committee and were in compliance with the guidelines of the Canadian Council on Animal Care.

Excitatory burst neurons (EBNs) and inhibitory burst neurons (IBNs) in the paramedian pontine reticular formation were first identified, in head-restrained animals, on the basis of the following: (1) their anatomical location [EBNs: $1-2 \mathrm{~mm}$ rostral to the abducens nucleus, $0.5-1.5 \mathrm{~mm}$ from the midline; IBNs: $0-2 \mathrm{~mm}$ caudal to the abducens nucleus, $0.5-1.5$ $\mathrm{mm}$ from the midline; both areas correspond to previous anatomical characterizations (Strassman et al., 1986)], (2) their characteristic "burst" discharge during horizontal saccades in the ipsilateral direction, and (3) the absence of modulation during slow phases of vestibular nystagmus [to distinguish SBNs from "burst-driver" neurons that have been reported in a similar region in cat (Bergeron and Guitton, 2002)]. Similarly, neurons were identified as omnipause neurons (OPNs) on the basis of (1) their anatomical location, (2) their characteristic "pause" discharge during saccades in all directions, and (3) the absence of modulation during vestibular slow phases (Strassman et al., 1987).

After a neuron had been identified in the head-restrained condition, the monkey's head was carefully released from its restraint. The monkey was then able to rotate its head through the natural range of motion in the yaw (horizontal), pitch (vertical), and roll (torsional) axes. Monkeys made horizontal gaze shifts of variable magnitudes (amplitudes: 20, 40, and $60 \mathrm{deg}$, with no vertical elevation) to track the laser target as it was stepped across the midline. To minimize the occurrence of anticipatory gaze shifts, the interval of fixation before target stepping was randomly varied between 800 and $1500 \mathrm{~ms}$. During a given experimental session, monkeys made gaze shifts of each amplitude; however, perturbations were applied for only during 40 deg gaze shifts. Head perturbations were randomly applied in $<15 \%$ of these trials, so that the remaining $85 \%$ of the gaze shifts of this amplitude were unperturbed (i.e., control gaze shifts). The short duration perturbations were applied using a programmable torque motor (model 2320; Animatics, Santa Clara, CA) that was securely coupled to the animal's skull through precision universal joints (Huterer and Cullen, 2002). Constant torque pulses (6 newton meter) were applied for $10 \mathrm{~ms}$ in the direction opposite to the ongoing gaze shift (Fig. 1). The resulting perturbations were highly stereotyped (Fig. 1, inset). They began 30-40 ms after gaze shift onset, had durations of $20-30 \mathrm{~ms}$, peak head velocities of $100-150 \mathrm{deg} / \mathrm{s}$, and peak accelerations of 10,000-15,000 $\mathrm{deg} / \mathrm{s}^{2}$, and generated total head displacements of $2-4 \mathrm{deg}$.

To get sufficient data for the analysis of neuronal responses during perturbed gaze shifts, it was necessary to identify a neuron based on the criteria that conformed to previous descriptions of OPNs, IBNs, and EBNs in head-restrained monkey, to then preserve isolation after the transition from head-restrained to head-unrestrained recording, to next collect control data during normal gaze shifts, and finally to maintain

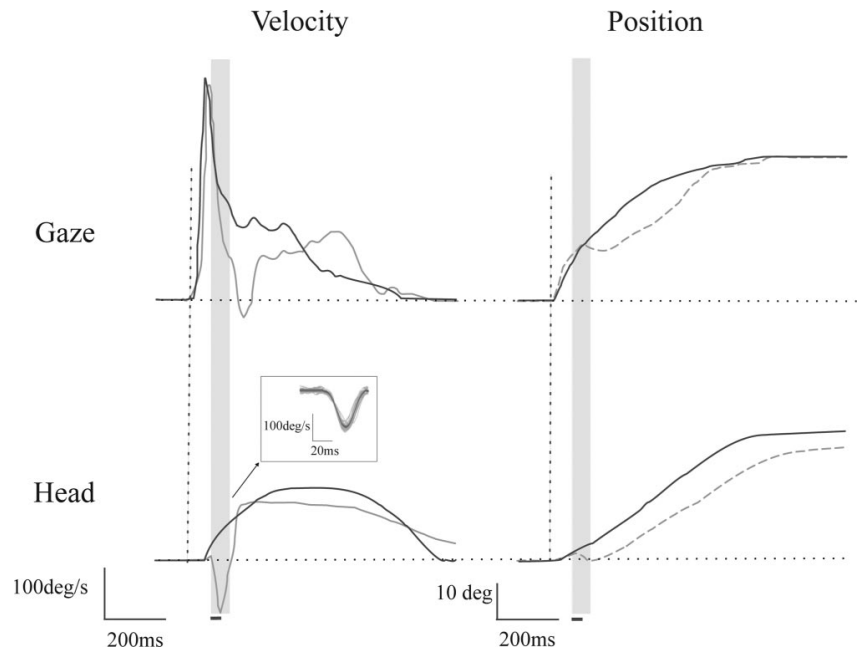

Figure 1. Example gaze and head velocity (left) and position (right) trajectories made during control gaze (black traces) and gaze shifts during which a torque motor was used to generate head perturbations in the direction opposite to that of the ongoing gaze (gray traces). The interval over which the torque pulse was applied is denoted by the horizontal bars. Shaded vertical bars indicate the short duration of the resultant head perturbation. Following the head perturbation, head velocity resumed in the direction of the gaze shift. Inset, The head velocity evoked in response to the same perturbations when the head was stationary. The average response (black trace) is superimposed on traces from 40 individual trials.

isolation during perturbed gaze shifts. Maintaining isolation of neurons during the perturbed gaze shift experiment was particularly challenging because the perturbations reached accelerations of $15,000 \mathrm{deg} / \mathrm{s}^{2}$. Thus, it was considerably more difficult to maintain neuronal isolation during this paradigm than during control gaze shifts.

Data analysis was performed in the Matlab environment (The Mathworks, Natick, MA). Control and perturbed gaze shifts for a given target presentation were searched for best possible matches of eye and head dynamics before perturbation onset. The "best-match" control gaze shift was obtained using a least-squared algorithm, and was visually verified. Gaze, eye, and head-movement onsets were defined using a $20 \mathrm{deg} / \mathrm{s}$ criterion. Perturbation onset was detected using a $1500 \mathrm{deg} / \mathrm{s}^{2}$ head acceleration criterion. For OPNs, pause onset and offset were marked as the time of the first spike (sp) encountered while scanning backward or forward in time from peak gaze velocity, respectively. Neurons were categorized as SBNs if the mean period between the onset of the first spike and the onset of eye velocity was $\leq 15 \mathrm{~ms}$ (Scudder et al., 1988; Cullen and Guitton, 1997).

SBN firing rates were represented as a spike density function in which a Gaussian function (SD of $5 \mathrm{~ms}$ ) was convolved with the spike train of the neuron (Cullen et al., 1996). Discharge perturbation onset was defined as the intersection of two regression lines, the first estimated on the firing rate curve 1-10 ms before perturbation onset, the second estimated $5-10 \mathrm{~ms}$ after perturbation onset. Discharge modulations were analyzed during saccades, control, and perturbed gaze shifts using dynamic analysis techniques (Cullen et al., 1996; Cullen and Guitton, 1997).

The model structure used during eye-only saccades was $\operatorname{FR}\left(t-t_{\mathrm{a}}\right)=b$ $+r \dot{E}(t)$, where $\operatorname{FR}(t)$ and $\dot{E}(t)$ are the instantaneous firing rate and eye velocity, " $b$ " is a bias term, and " $r$ " is the eye velocity sensitivity of a neuron. During control and perturbed gaze shifts, the model was modified to the following: $\operatorname{FR}\left(t-t_{\mathrm{d}}\right)=b+r \dot{E}(t)+g \dot{H}(t)$, where $\dot{H}(t)$ is the instantaneous head velocity, and " $g$ " is the head velocity sensitivity of the neuron. Model parameters were estimated on the data using a leastsquare algorithm, and the dynamic lead time of the neuron $\left(t_{\mathrm{d}}\right)$ was estimated during head-restrained saccades (Cullen et al., 1996). Goodness-of-fits were quantified using the variance-accounted-for $[\mathrm{VAF}=1-[\operatorname{var}(\bmod -\mathrm{fr}) / \operatorname{var}(\mathrm{fr})]$, where mod represents the modeled firing rate and fr represents the actual firing rate; perfect fits yield VAF $=$ 1]. Probability distributions of the model parameters were estimated using a nonparametric bootstrap approach (Richmond et al., 1987; Sokal 
and Rohlf, 1995; Press et al., 1997; Carpenter and Bithell, 2000; Sylvestre and Cullen, 2002).

To describe neuronal responses during control and perturbed gaze shifts, a model structure that incorporated two delays was also tested: $\mathrm{FR}(t)=b+r_{1} \dot{\mathrm{E}}\left(t+t_{\mathrm{d} 1}\right)+\mathrm{g}_{1} \dot{\mathrm{H}}\left(t+t_{\mathrm{d} 1}\right)+r_{2}$ $\dot{E}\left(t-t_{\mathrm{d} 2}\right)+g_{2} \dot{\mathrm{H}}\left(t-t_{\mathrm{d} 2}\right)$, where $t_{\mathrm{d} 1}$ and $t_{\mathrm{d} 2}$ represent a neuron's feedforward and feedback connectivity, respectively. Although optimization of this model required the estimation of an additional three parameters, it on average produced only a $\sim 4-5 \%$ increases in VAF. In addition, the simpler one-delay model provided more robust parameter estimates. As a result, for the purposes of the present study, in which the main goal was to demonstrate that the same model can describe both the control and perturbed gaze shifts, we used a one-delay model, which approximates the two-delay model when the feedforward and feedback delays are relatively small. This approximation is consistent with the short delays measured previously between SBN discharge and movement (Cullen and Guitton, 1997) as well as between headmovement feedback and changes in neuronal response in the present report.

\section{Results}

Brainstem premotor excitatory and inhibitory burst neurons (EBNs and IBNs; collectively, saccadic burst neurons, SBNs) drive ipsilateral and inhibit contralateral extraocular motoneurons, respectively, to generate rapid horizontal saccades. The activity of SBNs is in turn gated by inhibi-

tory projections from omnipause neurons (OPNs) when saccades are unnecessary. In the present study, the single-unit activity of all OPNs $(n=17)$ and SBNs $(n=26 ; n=16$ and 10 , IBNs and EBNs) was recorded during head-restrained saccades, headunrestrained gaze shifts and perturbed gaze shifts (see Materials and Methods). Consistent with previous reports, the duration of OPN pauses and SBN bursts was tightly correlated with saccade duration in the head-restrained condition (Paré and Guitton, 1998; Phillips et al., 1999) and with gaze movement duration when the head was released and monkeys used coordinated eyehead movements to redirect their gaze (gaze shifts) (Everling et al., 1998; Paré and Guitton, 1998; Phillips et al., 1999). The black traces in Figure 2 illustrate data from a typical OPN and SBN during 40 deg control gaze shifts. In Figure 2, $B$ and $C$, cell discharges have been aligned on gaze shift onset. Immediately before gaze shift onset, the OPN paused, while the SBN began to burst. In Figure 2, $D$ and $E$, the same trials have been aligned on gaze shift end. The offset of the OPN pause and SBN burst was tightly coupled to gaze shift offset.

The feedback signals that drive SBNs and OPNs during gaze shifts were probed by experimentally altering movement trajectories; mid-flight perturbations were applied to the head while neuronal responses were simultaneously recorded. Perturbations were applied at random on a minority of trials $(<15 \%$; see Materials and Methods), so that the monkey could not predict when they would occur. Each perturbation was applied $\approx 40 \mathrm{~ms}$ after gaze shift onset and was relatively short in duration such that it constituted only $\sim 10 \%$ of the duration of a perturbed gaze shift (Fig. 2A, gray traces). As observed previously, perturbed gaze shifts remained accurate following head perturbations ( $p=0.46$;
B.

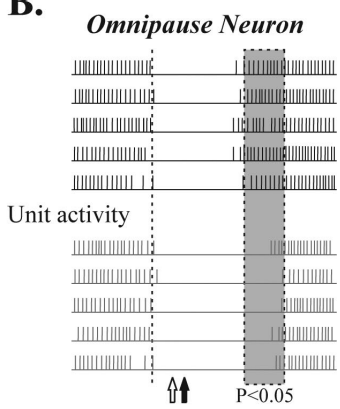

$\overline{100 \mathrm{~ms}}$

C.

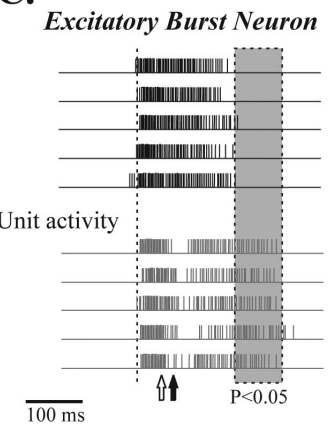

D.

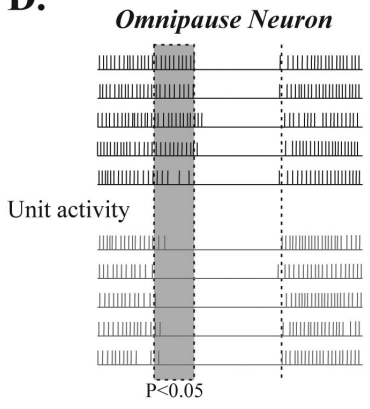

$\overline{100 \mathrm{~ms}}$

E.

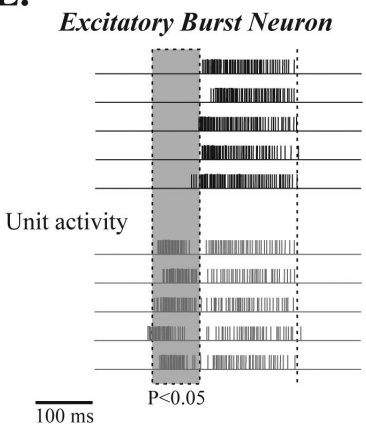

Figure 2. Head perturbation effects on gaze shift and neuronal responses. $\boldsymbol{A}$, Matched control (black) and perturbed (gray) shifts (see Materials and Methods). Vertical dashed line, gaze onset; shaded box, average increase in perturbed trials were aligned on gaze shift onset and offset, respectively. $\boldsymbol{C}, \boldsymbol{E}$, Example EBN activity recorded during the control and perturbed trials shown in $\boldsymbol{A}$. Traces were aligned on gaze shift onset and offset, respectively.

paired $t$ test, matched perturbed vs control trials) (Laurutis and Robinson, 1986; Guitton and Volle, 1987; Tomlinson, 1990; Tabak et al., 1996; Cullen et al., 2004) Importantly, the applied transient head perturbations did not completely interrupt the gaze shift (i.e., gaze velocity was not driven to zero velocity for a sustained time interval). Rather, they modified ongoing gaze shifts in two critical ways: (1) gaze shift durations increased significantly $(p<0.05)$ (Fig. $2 A$, gray box), and (2) the dynamics of the resultant gaze and eye movements were considerably altered (Fig. 2A, compare black and gray curves). Below, we assess how these two effects were reflected in the responses of OPNs and SBNs.

\section{Neural correlates of increased gaze shift duration}

The gray traces in Figure 2 illustrate recordings made from a representative OPN (Fig. 2B,D) and SBN (Fig. 2C,E) during perturbed gaze shifts. The onset of OPN and SBN saccade-related activity (before the perturbation) remained tightly coupled to gaze shift onset at all times (Fig. 2B,C). The current experimental design allowed us to make specific predictions regarding the offset of neuronal responses during perturbed trials (see Introduction). If two completely independent premotor feedback circuits specified the eye and head movements, SBN and OPN (i.e., elements of the eye premotor circuitry) responses should remain unaffected by the head perturbation. Instead, we observed a substantial increase in the duration of OPN pauses and SBN bursts. Notably, the offset of all OPN and SBN responses during perturbed trials remained tightly synchronized to gaze shift offset, updating in parallel with the resultant increase in movement duration (Fig. $2 D, E$ ). This finding that neurons are updated "online" as a function of the artificially modified head-movement 

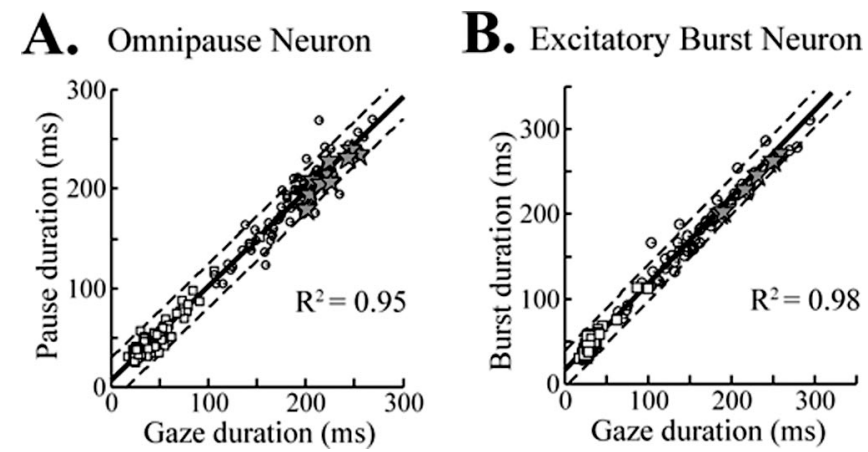

Figure 3. Following perturbations, neural responses were updated on-line to remain tightly correlated with gaze shift duration. $\boldsymbol{A}$, Correlation between movement and pause duration for the example OPN. Squares, circles, and stars indicate data from eye-only saccades, control, and perturbed gaze shifts, respectively. $\boldsymbol{B}$, Correlation between movement and burst duration for the example EBN. Note: the pause and burst duration of OPNs and SBNs were well related to saccade duration in the head-restrained condition (mean slope, $1.06 \pm 0.40,0.97 \pm 0.40$, $1.04 \pm 0.20$; mean $r=0.78 \pm 0.14,0.79 \pm 0.20$, and $0.78 \pm 0.21$; for OPNs, EBNs, and IBNs, respectively) and to control gaze shift duration in the head-unrestrained condition (mean slope, $1.00 \pm 0.30,1.00 \pm 0.14$, and $1.00 \pm 0.20 ;$ mean $r=0.78 \pm 0.19,0.74 \pm 0.21$, and $0.82 \pm$ 0.20 ; for OPNs, EBNs, and IBNs, respectively).

feedback signals is consistent with models that use a single integrated comparator to control gaze shifts.

To quantify the lengthening of saccade-related responses, we compared the duration of the pause of each neuron or burstrelated activity to gaze shift duration during perturbed gaze shifts. For all SBNs and for $94 \%$ of OPNs, the relationship between pause (OPNs) or burst (SBNs) duration and gaze shift duration was statistically indistinguishable during perturbed trials versus control gaze shifts and saccades (Fig. $3 A, B$, respectively) $(p=0.20-0.99, p=0.50-0.98, p=0.45-0.98$; OPNs, IBNs, and EBNs, respectively; $t$ test on the regression slopes of controls vs perturbed trials). Thus, neuronal responses remained similarly coupled to gaze movement duration across all conditions.

To evaluate how well, at the neuronal population level, perturbation-induced increases in gaze shift durations were represented by changes in response durations, an updating index (UI) (see legend of Fig. 4) was calculated for each unit. A UI value of zero indicated that the duration of neuronal discharges did not change during perturbed versus control trials, but a value of one indicated that increases in discharge duration were identical to increases in movement duration (i.e., perfect updating). The distributions of UI values are shown in Figure $4 A-C$. On average, the discharge duration of all three populations of cells displayed nearly perfect updating in response to head perturbations so that the overall distribution was centered near unity (Fig. 4D). These results confirm that the command generated by the saccadic premotor circuitry is altered in real time, by means of dynamic feedback, to account for the head-movement trajectory that is actually accomplished during a gaze shift.

\section{Neural correlates of altered gaze shift dynamics}

The application of transient head perturbations not only increased gaze shift duration but also altered the dynamics of the resultant gaze and eye trajectories (Fig. 2A). There were three main differences in the dynamics of perturbed and control gaze shifts. First, as observed previously (Huterer and Cullen, 2002; Cullen et al., 2004), a compensatory eye movement (i.e., in the direction opposite to the head perturbation) immediately followed perturbation onset (within 5-6 ms) (Fig. 2A, small arrow). This initial behavioral response has been comprehensively de-
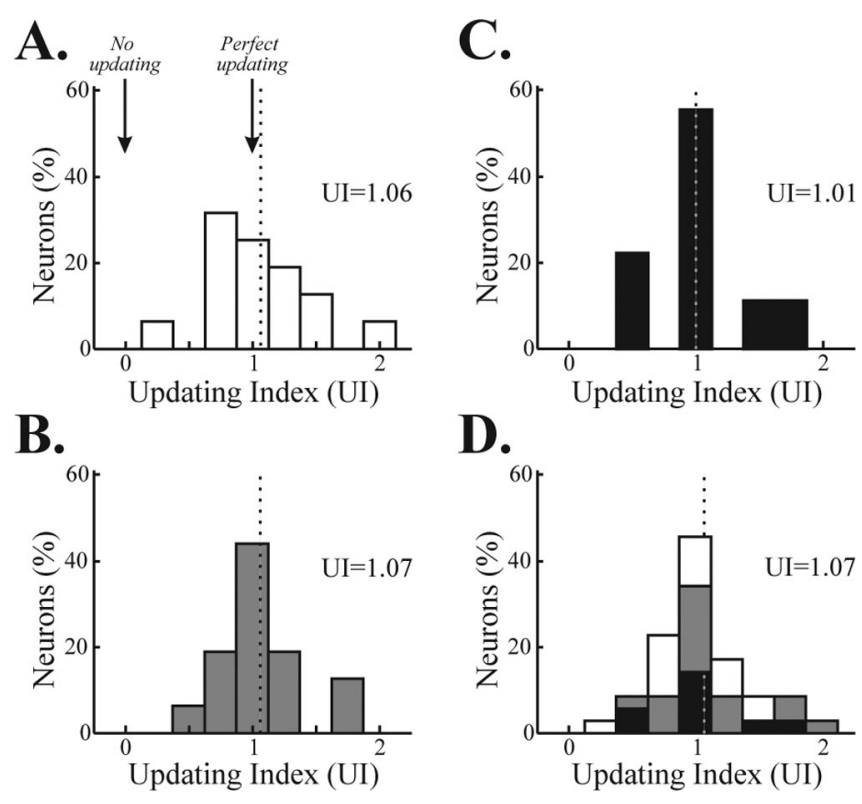

Figure 4. Distribution of Uls. We define Ul as (mean increase in discharge duration)/(mean increase in movement duration), calculated for matched perturbed versus control trials; zero indicates no updating, and one indicates perfect updating. Average Uls are indicated for each panel. $\boldsymbol{A}$, Distribution for OPNs. The vertical dotted line indicates the average UI. $\boldsymbol{B}$, Distribution for IBNs. C, Distribution for EBNs. D, Combined distribution for all neurons. The different neuron types are shown using the color scheme from $\boldsymbol{A}-\boldsymbol{C}$.

scribed previously and shows that the vestibulo-ocular reflex (VOR) is functional but attenuated early in a gaze shift. Subsequently, we consistently observed an abrupt decrease in gaze velocity (Fig. $5 A$, black area, top inset), which was then followed by a reacceleration that resulted in a late increase in gaze velocity relative to control gaze shifts (Fig. 5A, black area, bottom inset).

The discharges of SBN firing showed changes during perturbation trials that clearly mirrored these altered gaze dynamics. As compared with control trials, neuronal responses showed a relative inhibition (Fig. $5 B$, black area, top inset) subsequent to the perturbation, followed by a reactivation that outlasted responses during control gaze shifts (Fig. 5B, black area, bottom inset). Moreover, we examined the latency between neuronal and behavioral changes in dynamics and found that neurons responded to gaze shift perturbations at a very short latency. Figure 6 shows responses during control versus perturbed gaze shifts of the example $\operatorname{EBN}(A, B)$ as well as an example $\operatorname{IBN}(C, D)$ with the time scales further expanded for perturbed gaze shifts. The nearly instantaneous updating of neuronal responses to head perturbations is evident as a sudden decrease in activity immediately following the perturbation. On average, the latency of the neuronal response relative to the onset of the head velocity perturbation was $3.8 \pm 2.8 \mathrm{~ms}$ (Fig. $6 E$ ) (see Materials and Methods). To further examine the specific mechanism responsible for updating neuronal response following perturbations, we applied a multiple regression model to quantify the firing rate dynamics of SBNs (Cullen et al., 1996). In agreement with previous reports (Van Gisbergen et al., 1981; Cullen and Guitton, 1997), each SBN was found to encode eye velocity during head-restrained saccades $[$ mean variance-accounted-for $[\mathrm{VAF}]=0.61 \pm 0.15$ and $0.53 \pm$ 0.18 , IBNs and EBNs; VAF $=1$ indicates a perfect goodness-offit]. In addition, as shown in Figure 7, $A$ and $B$, the same eyebased model that described a neuron during head-restrained saccades could not be used to predict its response during control or perturbed gaze shifts $(\mathrm{VAF}=0.15 \pm 0.76)$. This confirms that the 


\section{A. Gaze velocities Average gaze velocities}

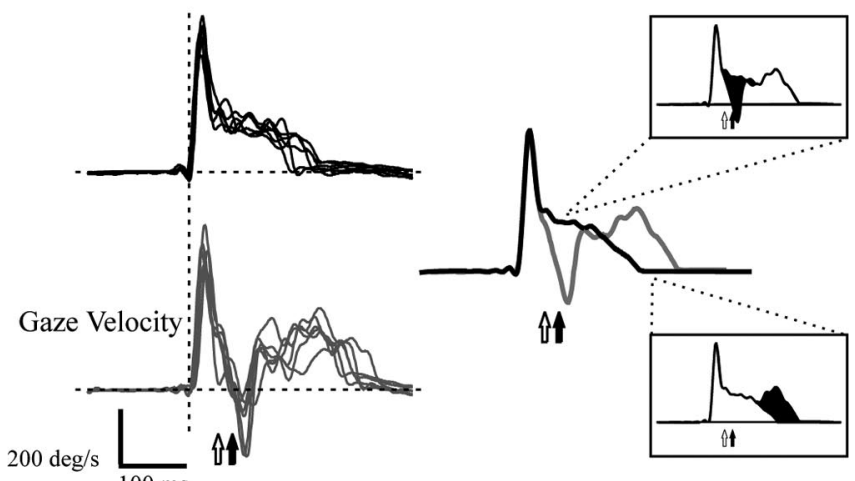

B.

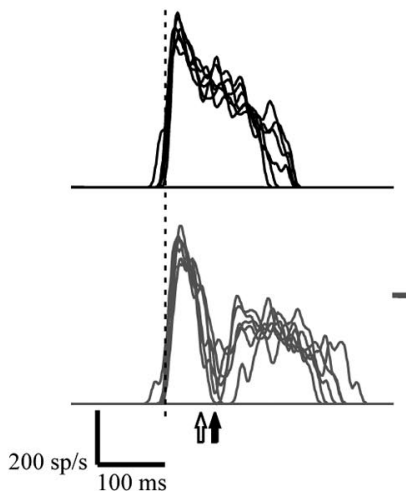

Average firing rates

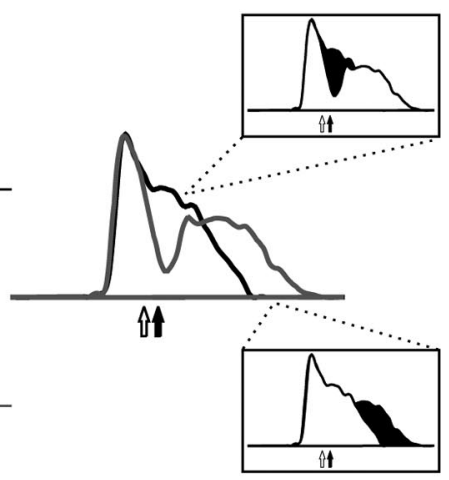

Figure 5. Changes in the instantaneous firing rates of SBNs mirrored gaze shift dynamics after head perturbations. Left, Example gaze velocity $(\boldsymbol{A})$ and firing rate traces $(\boldsymbol{B})$ for the example EBN during the control (black traces) and perturbed (gray traces) trials shown in Figure 1. Right, Average gaze velocity and firing rate traces computed from the control and perturbed traces shown to the left. Insets, Differences between the average gaze velocity and firing rate traces (black areas). The top insets illustrate the transient decrease in gaze velocity and firing rate immediately after perturbations, and bottom insets emphasize the overall increase in duration.

relationship between neuronal responses and movement trajectories during gaze shifts changed significantly (Cullen et al., 1993; Cullen and Guitton, 1997; Ling et al., 1999).

During gaze shifts, the extraocular motoneurons receive inputs not only from SBNs but also from the head-velocitysensitive neurons in the vestibular nuclei that mediate the VOR (Roy and Cullen, 1998). Because the head velocity signal carried to motoneurons by these vestibular neurons must be offset (Cullen et al., 2000), it is logical that SBNs might encode head as well as eye velocity signals during gaze shift. Indeed, consistent with our previous findings (Cullen et al., 1993; Cullen and Guitton, 1997), regression models that included both eye and head coefficients markedly improved fits to SBN responses (VAF $=$ $0.57 \pm 0.18)$. The estimated head-velocity coefficients were significant and excitatory for most (72\%) SBNs ( $\mathrm{g}=0.4 \pm 0.6$ $\mathrm{sp} / \mathrm{deg} ; \mathrm{r}=0.5 \pm 0.3 \mathrm{sp} / \mathrm{deg} ; \mathrm{b}=175 \pm 100 \mathrm{sp} / \mathrm{s}$; see Materials and Methods). Subsequently, we examined whether the responses of a neuron during perturbed gaze shifts could be accurately predicted using the model estimated during control trials. We found that the same regression model could be used to fit both control and perturbed gaze shifts (compare Fig. $7 C-D)(\mathrm{VAF}=0.50 \pm$ 0.21 ), and that when the regression coefficients were separately estimated for each condition they were statistically identical ( $p=$
A.

B.
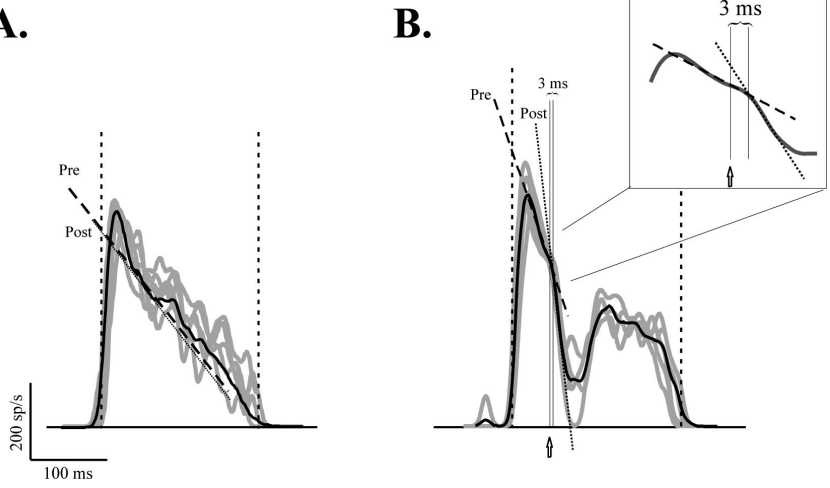

C.

D.
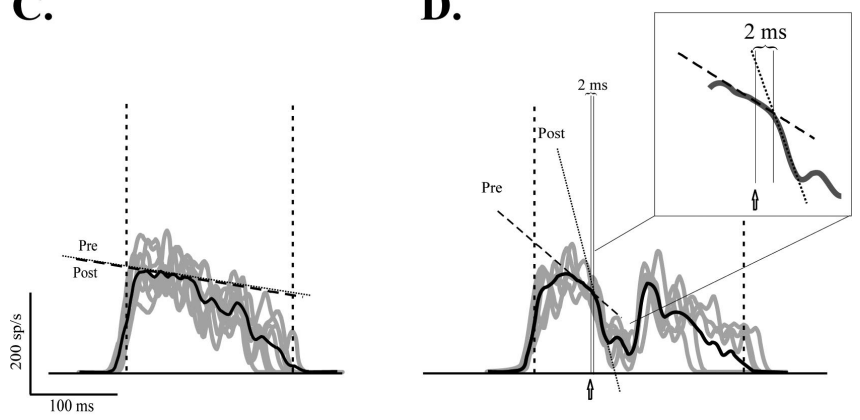

E.

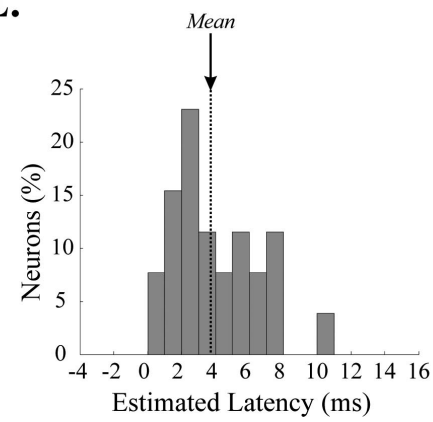

Figure 6. Responses of the example EBN $(\boldsymbol{A}, \boldsymbol{B})$ and an example IBN $(\boldsymbol{C}, \boldsymbol{D})$ during control versus perturbed gaze shifts. Responses from individual trials (gray traces) and average responses (black traces) are superimposed, and dashed vertical lines denote the onset and offset of the gaze shift. B, D, Small arrows indicate the onset of the head velocity perturbation. Dashed and dotted lines indicate the best fit to the response of the unit before and after the perturbation, respectively. The gray vertical lines denote the interval between the onset of the perturbation and the intersection of the lines of best fit. The example neurons were typical in that their modulation was attenuated at very short latencies ( 3 and $2 \mathrm{~ms}$ ) in response to applied head perturbations. $A, C$, Control data for which the timing of the head perturbation in perturbation trials $(\boldsymbol{B}, \boldsymbol{D})$ was used as a reference to produce the lines of best fit to the unit's response. Inset, Time scales of the mean response and best line fits have been further expanded near the onset of the perturbation. $\boldsymbol{E}$, Distribution of response latency across the population of SBNs. The vertical dotted line indicates the average response latency. Pre, Before perturbation; Post, after perturbation.

$0.19,0.38,0.50,0.38 ; \mathrm{b}, \mathrm{r}, \mathrm{g}$, and VAF, paired $t$ test). Therefore, the activity of SBNs retained a constant relationship with head velocity during perturbed and control gaze shifts, indicating that their responses were dynamically updated by the same head-related information in both conditions.

\section{Discussion}

Feedback and the control of gaze shifts

We have shown that the premotor neurons that command saccadic eye movements in the head-restrained condition are up- 


\section{A. Saccadic Prediction (control)}
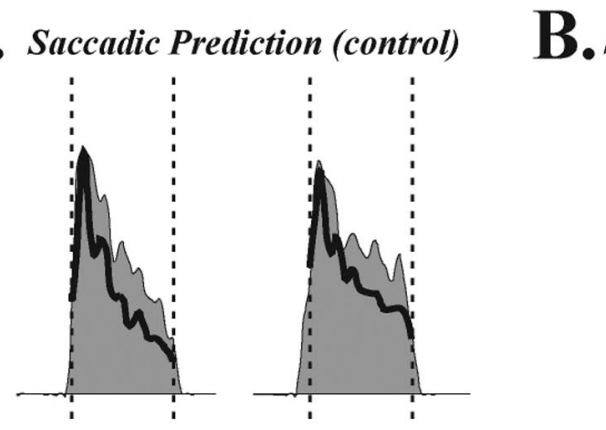

Saccadic Prediction (perturbed)

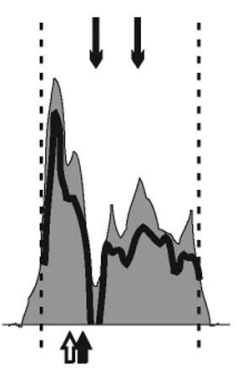

\section{C.Eye+Head Estimation (control)}
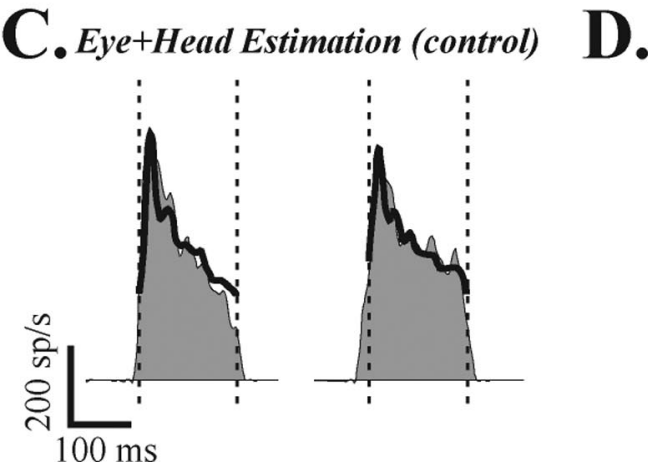

Eye+Head Prediction (perturbed)

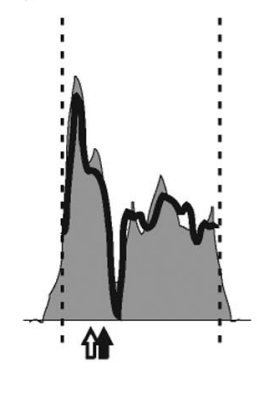

Figure 7. SBNs dynamically encoded the same eye and head-movement-related modulations during control and perturbed gaze shifts. Predictions of the discharges of a neuron during control gaze shifts $(\boldsymbol{A})$ and perturbed gaze shifts $(\boldsymbol{B})$, obtained using an eye-based model (see Materials and Methods) estimated during eye-only saccades. Model predictions (thick curve) are shown superimposed on the firing rate (gray shaded area) for the example EBN. Downward arrows indicate time intervals at which the fits to the data were particularly poor. C, Model fits to the firing rate of the same neuron, obtained using a different model in which eye and head velocity coefficients were estimated (see Materials and Methods) from control gaze shifts. Note the marked improvement in goodness-of-fit. $\boldsymbol{D}$, Very good model predictions were obtained when the model estimated during control trials $(\boldsymbol{C})$ was applied to the firing rate of the neuron recorded during perturbed gaze shifts.

dated on-line by head-movement-related feedback during coordinated eye-head gaze shifts. First and most importantly, perturbation-induced increases in gaze shift durations were accompanied by equivalent changes in response durations of OPNs and SBNs, such that neuronal activity remains tightly synchronized to gaze shift offset. Second, SBNs discharges were dynamically updated in response to head perturbations; the relationship between neural activity and head velocity remained comparable during perturbed and control gaze shifts, indicating that the head-related feedback was comparable in both conditions. Together, our findings show that on-line feedback of headmovement updates the durations and dynamics of neurons in the saccadic premotor network to preserve global movement accuracy during gaze shifts.

Our findings address an important question in motor control; namely, how is accuracy ensured during behaviors that are accomplished by the movement of multiple body segments? This has been a controversial issue with respect to eye-head coordination during gaze shifts, and two general classes of models had been proposed to account for the available data: gaze feedback models (Tomlinson 1990; Galiana and Guitton, 1992; Goossens and Van Opstal, 1997) and separate feedback models (Phillips et al., 1995; Freedman, 2001; Sparks et al., 2002). In the first class of models, gaze accuracy is maintained by comparing the desired change in gaze position with the actual gaze displacement, which is calculated from feedback of the ongoing eye and head movements. The difference between desired and actual gaze displace-
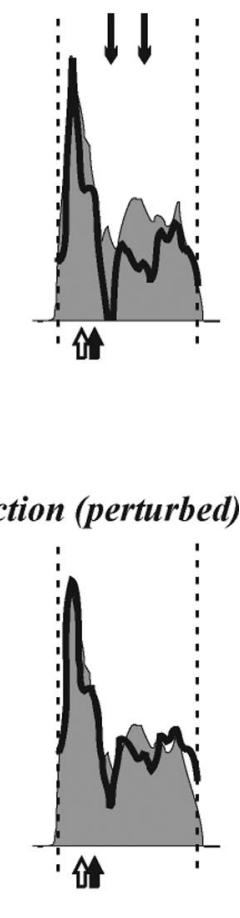

ment is calculated throughout the course of a gaze shift to provide a dynamic gaze feedback signal, which drives the eye and head premotor circuits until it is nulled (i.e., when gaze lands on target). In the second class of models, the desired change in gaze is decomposed to drive two separate controllers, so that two feedback loops separately compute the desired changes in eye and head position. In this model structure, the input to the saccadic burst generator (i.e., OPNs and SBNs) encodes the desired position of the eye at gaze shift onset.

The results of previous studies had proven ambiguous with respect to both classes of models. Numerous studies have shown that gaze accuracy is unaffected by the passive application of head perturbations during an ongoing gaze shift (Tomlinson and Bahra, 1986; Pelisson et al., 1988; Tabak et al., 1996). Dynamic gaze feedback models incorporate this experimental result using their single feedback loop. Separate feedback models rely on either neck reflexes (Freedman, 2001; Freedman and Quessy, 2004; Quessy and Freedman, 2004) or, alternatively, the precise titration of the VOR (Phillips et al., 1995) to ensure gaze accuracy. Similarly, the results of single-unit recording experiments (Cullen et al., 1993; Cullen and Guitton, 1997; Phillips et al., 1999) have been used to support either class of model. Although the activity of extraocular motoneurons is well predicted by means of the same simple linear relationship with eye movement during head-restrained saccades and coordinated eye-head movements (Sylvestre and Cullen, 1999; Cullen et al., 2000), SBNs encode significant head-velocity-related information during gaze shifts (Cullen and Guitton, 1997; Ling et al., 1999). A limitation inherent to these neurophysiological studies was that the responses of SBNs could only be characterized from a downstream perspective (i.e., correlations between neuronal activity and the resultant eye, head and/or gaze movements). Any inputs to motoneurons from other premotor neurons, for example from the vestibulo-ocular reflex pathways, would have altered the relationship between the executed eye movement and that commanded by the recorded neuron. As a result, it is not possible to make inferences about the nature of the upstream command signals based on previous results (Cullen and Guitton, 1997; Sparks, 1999).

Here, with the limitations of previous approaches in mind, we developed a new approach to assess the discharge properties of premotor neurons during gaze shifts. Transient head perturbations were introduced during natural gaze shifts, to characterize saccadic premotor neurons from a feedback rather than feedforward perspective. Our results are consistent with a theoretical framework in which gaze shift accuracy is ensured by a combined gaze comparator. The structure of this model predicts that the drive to both eye and head premotor circuits should be updated in response to a head perturbation to preserve gaze accuracy. Indeed, this is exactly what we observed. In contrast, models in which gaze shift accuracy is ensured by separate controllers, specifying a priori the amplitude of eye and head are difficult to rec- 
oncile with the present results. These models do not predict that the gating and control of saccades by OPNs and SBNs, respectively, should be altered to reflect the changes in gaze shift dynamics that result from experimentally applied head-movement perturbations.

The correspondence between the idea of an integrative gaze comparator and that of optimal feedback control, which has been proposed to explain the neural control of limb movements (Todorov and Jordan, 2002), is noteworthy. A principal feature of both models is that variability in movement trajectories is not problematic (Bizzi et al., 1984; Flash and Hogan, 1985; Harris and Wolpert, 1998), because the end goal of the movement is achieved as a result of afferent feedback. In addition, these models can account for well described in-flight corrections of movement trajectories following perturbations of both systems [limb (Todorov and Jordan, 2002; Kording and Wolpert, 2004); gaze (Guitton et al., 2003)]. At the neuronal level, the present results clearly demonstrate the convergence of oculomotor and headmovement-related afferent information up to the final stages of premotor processing in the gaze control system. This headrelated afferent signal modifies neuronal responses in a manner that is consistent with the goal of gaze shift. The integration of oculomotor and head-movement feedback on premotor neurons is consistent with the proposal that the gaze control system is designed to guarantee accurate gaze shifts rather than enforce a specific eye or head trajectory. Similarly, for the limb control system, previous studies have shown that neurons in the primary motor cortex (area M1) respond to afferent feedback resulting from passive movements of multiple joints (Wong et al., 1978; Scott and Kalaska, 1997) and cutaneous stimulation (Strick and Preston, 1978; Lemon, 1981). It is likely that these sensory inputs mediate the dynamic sensory feedback required to account for the rapid (i.e., as short as $20 \mathrm{~ms}$ ) responses of $\mathrm{M} 1$ neurons to limb perturbations during hand stabilization (Wolpaw, 1980). However, a challenge of future work will be to develop theoretical approaches to explicitly assess whether the gaze and limb control systems integrate feedback signals in an optimal manner (for review, see Scott, 2004).

Previous reports have provided evidence that the combined comparator that controls gaze shifts might be centered on the superior colliculus (Choi and Guitton, 2002; Matsuo et al., 2004) and/or cerebellum (Quaia et al., 1999). However, because the mechanical (Choi and Guitton, 2002; Matsuo et al., 2004) or electrical (Quaia et al., 1999) perturbations used in these previous studies interrupted gaze movements (i.e., rendered gaze stable for hundreds of milliseconds), it has not been possible to resolve whether the effects of the perturbations represented dynamic corrections to the ongoing gaze shift or the programming of a second corrective movement. In contrast, the relatively short time frame of the effects described here and the absence of gaze shift interruptions rule out the central programming of a corrective movement. Moreover, perturbations were applied infrequently, so that the monkey could not predict when they would occur. The present report therefore provides the first conclusive demonstration of integrated feedback control during gaze shifts.

\section{Implications for brainstem premotor processing}

At first glance, it may appear surprising that SBN neurons encode head as well as eye movement information because they project to extraocular and not to neck motoneurons. However, the headmovement-related response that we observe during gaze shifts can be accounted for by known brainstem mechanisms (Cullen and Guitton, 1997; Roy and Cullen, 1998; Sparks, 1999). Neurons in the medial vestibular nuclei, which mediate the direct vestibulo-ocular pathways, project directly to extraocular motoneurons. The head velocity signal carried by these vestibular neurons is attenuated, but not completely abolished, during gaze shifts (Roy and Cullen, 1998, 2002). The residual head velocity information carried by SBNs is therefore appropriate to offset this incoming vestibular-related drive, such that the responses of the extraocular motoneurons are solely related to eye motion during gaze shifts, as was shown previously (Cullen et al., 2000).

The existence of rapid response modulations at disynaptic or trisynaptic latencies suggests that, in addition to inputs from an upstream gaze comparator, a more direct nested source of headmovement-related feedback reaches the brainstem eye premotor circuitry. Indeed, a trisynaptic excitatory pathway has been described previously between the vestibular nerve and SBNs (Kitama et al., 1995). It has further been argued that this pathway, which is thought to play an important role in generating the quick phases of vestibular nystagmus, could function to facilitate eye movements during gaze shifts (Galiana and Guitton, 1992; Van Beuzekom and Van Gisbergen, 2002). Our results are consistent with this hypothesis at the single neuron level. Moreover, the proposal that the teleologically more ancient vestibular quickphase generator has been integrated within an upstream feedback controller is likely to have analogues in other motor control systems, illustrating an interesting example of convergent evolution. For example, during locomotion, there is evidence that spinal reflexes function in parallel with an upstream controller to adjust to perturbations (for review, see Donelan and Pearson, 2004). Our results highlight that the integration of existing (and rapid) reflex circuitries may be a generalized strategy for guaranteeing rapid and accurate motor control.

\section{References}

Bergeron A, Guitton D (2002) Multiple-step gaze shifts: omnipause (OPNs) and collicular fixation neurons encode gaze position error; OPNs gate saccades. J Neurophysiol 88:1726-1742.

Bernstein NI (1967) The coordination and regulation of movements. Oxford: Pergamon.

Bizzi E, Accornero N, Chapple W, Hogan N (1984) Posture control and trajectory formation during arm movement. J Neurosci 4:2738-2744.

Carpenter J, Bithell J (2000) Bootstrap confidence intervals: when, which, what? A practical guide for medical statisticians. Stat Med 19:1141-1164.

Choi WY, Guitton D (2002) Discharge characteristics of saccade-related neurons in primate superior colliculus during head-perturbed gaze shifts. Soc Neurosci Abstr 28:11.7.

Cullen KE, Guitton D (1997) Analysis of primate IBN spike trains using system identification techniques. II. Relationship to gaze, eye, and head movement dynamics during head-free gaze shifts. J Neurophysiol 78:3283-3306.

Cullen KE, Guitton D, Rey CG, Jiang W (1993) Gaze-related activity of putative inhibitory burst neurons in the head-free cat. J Neurophysiol 70:2678-2683.

Cullen KE, Rey CD, Guitton D, Galiana HL (1996) The use of system identification techniques in the analysis of oculomotor burst neuron spike train dynamics. J Comput Neurosci 3:347-368.

Cullen KE, Galiana HL, Sylvestre PA (2000) Comparing extraocular motoneuron discharges during head-restrained saccades and headunrestrained gaze shifts. J Neurophysiol 83:630-637.

Cullen KE, Huterer M, Braidwood DA, Sylvestre PA (2004) Time course of vestibuloocular reflex suppression during gaze shifts. J Neurophysiol 92:3408-3422.

Donelan JM, Pearson KG (2004) Contribution of sensory feedback to ongoing ankle extensor activity during the stance phase of walking. Can J Physiol Pharmacol 82:589-598.

Everling S, Paré M, Dorris MC, Munoz DP (1998) Comparison of the discharge characteristics of brain stem omnipause neurons and superior 
colliculus fixation neurons in monkey: implications for control of fixation and saccade behavior. J Neurophysiol 79:511-528.

Flash T, Hogan N (1985) The coordination of arm movements: an experimentally confirmed mathematical model. J Neurosci 5:1688-1703.

Freedman EG (2001) Interactions between eye and head control signals can account for movement kinematics. Biol Cybern 84:453-462.

Freedman EG, Quessy S (2004) Electrical stimulation of rhesus monkey nucleus reticularis gigantocellularis. II. Effects on metrics and kinematics of ongoing gaze shifts to visual targets. Exp Brain Res 156:357-376.

Freedman EG, Sparks DL (1997) Eye-head coordination during headunrestrained gaze shifts in rhesus monkeys. J Neurophysiol 77:2328-2348.

Galiana HL, Guitton D (1992) Central organization and modelling of eyehead coordination during orienting gaze shifts. Ann NY Acad Sci 656:452-471.

Goossens HH, Van Opstal AJ (1997) Human eye-head coordination in two dimensions under different sensorimotor conditions. Exp Brain Res 114:542-560.

Guitton D, Volle M (1987) Gaze control in humans: eye-head coordination during orienting movements to targets within and beyond the oculomotor range. J Neurophysiol 58:427-459.

Guitton D, Bergeron A, Choi WY, Matsuo S (2003) On the feedback control of orienting gaze shifts made with eye and head movements. Prog Brain Res 142:55-68.

Harris CM, Wolpert DM (1998) Signal-dependent noise determines motor planning. Nature 394:780-784.

Huterer M, Cullen KE (2002) Vestibuloocular reflex dynamics during highfrequency and high-acceleration rotations of the head on body in rhesus monkey. J Neurophysiol 88:13-28.

Kitama T, Ohki Y, Shimazu H, Tanaka M, Yoshida K (1995) Site of interaction between saccade signals and vestibular signals induced by head rotation in the alert cat: functional properties and afferent organization of burster-driving neurons. J Neurophysiol 74:273-287.

Kording KP, Wolpert DM (2004) Bayesian integration in sensorimotor learning. Nature 427:244-247.

Laurutis VP, Robinson DA (1986) The vestibulo-ocular reflex during human saccadic eye movements. J Physiol (Lond) 373:209-233.

Lemon RN (1981) Functional properties of monkey motor cortex neurones receiving afferent input from the hand and fingers. J Physiol (Lond) 311:497-519.

Ling L, Fuchs AF, Phillips JO, Freedman EG (1999) Apparent dissociation between saccadic eye movements and the firing patterns of premotor neurons and motoneurons. J Neurophysiol 82:2808-2811.

Matsuo S, Bergeron A, Guitton D (2004) Evidence for gaze feedback to the cat superior colliculus: discharges reflect gaze trajectory perturbations. J Neurosci 24:2760-2773.

Paré M, Guitton D (1998) Brain stem omnipause neurons and the control of combined eye-head gaze saccades in the alert cat. J Neurophysiol 79:3060-3076.

Pélisson D, Prablanc C, Urquizar C (1988) Vestibuloocular reflex inhibition and gaze saccade control characteristics during eye-head orientation in humans. J Neurophysiol 59:997-1013.

Phillips JO, Ling L, Fuchs AF, Siebold C, Plorde JJ (1995) Rapid horizontal gaze movement in the monkey. J. Neurophysiol 73:1632-1652.

Phillips JO, Ling L, Fuchs AF (1999) Action of the brainstem saccade generator during horizontal gaze shifts. I. Discharge patterns of omnidirectional pause neurons. J Neurophysiol 81:1284-1295.

Press WH, Teukolsky SA, Vetterling WT, Flannery BP (1997) Numerical recipes in C. The art of scientific computing, Ed 2, pp 689-693. New York: Cambridge, UP.

Quaia C, Lefevre P, Optican LM (1999) Model of the control of saccades by superior colliculus and cerebellum. J Neurophysiol 82:999-1018.

Quessy S, Freedman EG (2004) Electrical stimulation of rhesus monkey nucleus reticularis gigantocellularis. I. Characteristics of evoked head movements. Exp Brain Res 156:342-356.
Richmond BJ, Optican LM, Podell M, Spitzer H (1987) Temporal encoding of two-dimensional patterns by single units in primate inferior temporal cortex. I. Response characteristics. J Neurophysiol 57:132-146.

Roy JE, Cullen KE (1998) A neural correlate for vestibulo-ocular reflex suppression during voluntary eye-head gaze shifts. Nat Neurosci 1:404-410.

Roy JE, Cullen KE (2002) Vestibuloocular reflex signal modulation during voluntary and passive head movements. J Neurophysiol 87:2337-2357.

Scott SH (2004) Optimal feedback control and the neural basis of volitional motor control. Nat Rev Neurosci 5:532-546.

Scott SH, Kalaska JF (1997) Reaching movements with similar hand paths but different arm orientations. I. Activity of individual cells in motor cortex. J Neurophysiol 77:826-852.

Scudder CA, Fuchs AF, Langer TP (1988) Characteristics and functional identification of saccadic inhibitory burst neurons in the alert monkey. J Neurophysiol 59:1430-1454.

Scudder CA, Kaneko CS, Fuchs AF (2002) The brainstem burst generator for saccadic eye movements: a modern synthesis. Exp Brain Res 142:439-462.

Sokal RR, Rohlf FJ (1995) Biometry. The principles and practice of statistics in biological research, Ed 3, pp 820-825. New York: W. H. Freeman.

Sparks DL (1999) Conceptual issues related to the role of the superior colliculus in the control of gaze. Curr Opin Neurobiol 9:698-707.

Sparks DL, Barton EJ, Gandhi NJ, Nelson J (2002) Studies of the role of the paramedian pontine reticular formation in the control of head-restrained and head-unrestrained gaze shifts. Ann NY Acad Sci 956:85-98.

Strassman A, Highstein SM, McCrea RA (1986) Anatomy and physiology of saccadic burst neurons in the alert squirrel monkey. II. Inhibitory burst neurons. J Comp Neurol 249:358-380.

Strassman A, Ewinger C, McCrea RA, Baker RB, Highstein SM (1987) Anatomy and physiology of intracellularly labelled omnipause neurons in the cat and squirrel monkey. Exp Brain Res 67:436-440.

Strick PL, Preston JB (1978) Sorting of somatosensory afferent information in primate motor cortex. Brain Res 156:364-368.

Sylvestre PA, Cullen KE (1999) Quantitative analysis of abducens neuron discharge dynamics during saccadic and slow eye movements. J Neurophysiol 82:2612-2632.

Sylvestre PA, Galiana HL, Cullen KE (2002) Conjugate and vergence oscillations during saccades and gaze shifts: implications for integrated control of binocular movement. J Neurophysiol 87:257-272.

Tabak S, Smeets JB, Collewijn H (1996) Modulation of the human vestibuloocular reflex during saccades: probing by high-frequency oscillation and torque pulses of the head. J Neurophysiol 76:3249-3263.

Todorov E (2004) Optimality principles in sensorimotor control. Nat Neurosci 7:907-915.

Todorov E, Jordan MI (2002) Optimal feedback control as a theory of motor coordination. Nat Neurosci 5:1226-1235.

Tomlinson RD (1990) Combined eye-head gaze shifts in the primate. III. Contributions to the accuracy of gaze saccades. J Neurophysiol 64:1873-1891.

Tomlinson RD, Bahra PS (1986) Combined eye-head gaze shifts in the primate II. Interactions between saccades and the vestibuloocular reflex. J Neurophysiol 56:1558-1570.

Van Beuzekom AD, Van Gisbergen JAM (2002) Interaction between visual and vestibular signals for the control of rapid eye movements. J Neurophysiol 88:306-322.

Van Gisbergen JAM, Robinson DA, Gielen S (1981) A quantitative analysis of the generation of saccadic eye movements by burst neurons. J Neurophysiol 45:417-442.

Wolpaw JR (1980) Correlations between task-related activity and responses to perturbation in primate sensorimotor cortex. J Neurophysiol 44:1122-1138.

Wong YC, Kwan HC, MacKay WA, Murphy JT (1978) Spatial organization of precentral cortex in awake primates. I. Somatosensory inputs. J Neurophysiol 41:1107-1119. 